\title{
Effects of semantic cues on mathematical modeling: Evidence from word-problem solving and equation construction tasks
}

\author{
SHIRLEY A. MARTIN \\ University of Chicago, Chicago, Illinois \\ and \\ MIRIAM BASSOK \\ University of Washington, Seattle, Washington
}

\begin{abstract}
Mathematical solutions to textbook word problems are correlated with semantic relations between the objects described in the problem texts. In particular, division problems usually involve functionally related objects (e.g., tulips-vases) and rarely involve categorically related objects (e.g., tulips-daisies). We examined whether middle school, high school, and college students use object relations when they solve division word problems (WP) or perform the less familiar task of representing verbal statements with algebraic equations (EQ). Both tasks involved multiplicative comparison statements with either categorically or functionally related objects (e.g., "four times as many cupcakes [commuters] as brownies [automobiles]"). Object relations affected the frequency of correct solutions in the WP task but not in the EQ task. In the latter task, object relations did affect the structure of nonalgebraic equation errors. We argue that students use object relations as "semantic cues" when they engage in the sense-making activity of mathematical modeling.
\end{abstract}

Mathematics educators attempt to teach students that the formal rules of mathematics can be used to model real-life situations. To this end, they present students with word problems, such as the following: "Jane has 60 apples. She wants to place them in 5 baskets and have the same number of apples in each basket. How many apples should she place in each basket?" Word problems are short and highly contrived stories that describe quantitative relations between various objects (e.g., apples and baskets) and require mathematical solutions. To solve word problems correctly, students are expected to engage in mathematical modeling. First, they need to draw on their semantic and pragmatic knowledge in order to construct a situation model, or a representation of the refer-

The experiment reported here formed the basis of the first author's master's thesis at the University of Chicago under the supervision of M.B. and was supported by Royalty Research Fund Grant 65-3610 from the University of Washington to M.B. Parts of this research were presented at the 38th and 39th Annual Meetings of the Psychonomic Society, Philadelphia, PA, and Dallas, TX. We thank Lyn English and two anonymous reviewers for their helpful comments on a previously submitted draft. We also thank Katja Borchert, Amy Guthormsen, and John Miyamoto for helpful comments, discussions, and suggestions. Correspondence concerning this article should be addressed to either S. A. Martin, Conceptual and Historical Studies of Science, University of Chicago, 1126 E. 59th Street, Chicago, IL 60637 (e-mail: samartin@midway. uchicago.edu) or M. Bassok, Department of Psychology, University of Washington, Box 351525, Seattle, WA 98195 (e-mail: mbassok@u.washington.edu). ent problem situation (e.g., apples placed in baskets). Subsequently, they have to retrieve or construct an appropriate mathematical model (e.g., division) and instantiate it with the corresponding values from the situation model (e.g., 60 apples divided by 5 baskets). Although researchers differ in their specific proposals about the process of mathematical modeling (e.g., English \& Halford, 1995; Kintsch, 1988), they agree that students should draw on their world knowledge to constrain problem formulation, solution, and verification (Greeno, 1987).

Despite the intention of word problem designers, all too often students solve word problems correctly without engaging in modeling. This is because word problem texts provide students with standardized phrases and keywords that are highly correlated with correct solutions and therefore allow students to go directly "from words to equations" (Hinsley, Hayes, \& Simon, 1977). For example, students learn that the keyword altogether indicates that they should use addition (Nesher \& Teubal, 1975 ) or that times indicates that they should use multiplication (English, 1997). Because the correlation between standardized keywords and correct problem solutions is not perfect, however, reliance on such so-called translation cues can lead to systematic errors. For example, Clement, Lochhead, and Soloway (1979) asked engineering students to construct an algebraic equation to represent the multiplicative comparison statement "There are six times as many students $(S)$ as professors $(P)$." A 
substantial proportion of the participants committed "reversal errors," writing " $6 S=P$ " instead of " $6 P=S$." One reason for such reversal errors is that students translate the keyword times into multiplication and follow the order of the arguments in the verbal statement (Clement, 1982).

Reliance on translation cues to solve mathematical word problems is a less effortful process than modeling, if only because modeling requires nontrivial semantic and pragmatic inferences about the situation described in the problem text (e.g., Baranes, Perry, \& Stigler, 1989; Greer, 1993; Hinsley et al., 1977; Reusser, 1988; Verschaffel, De Corte, \& Lasure, 1994). Furthermore, reliance on translation cues does not require understanding of relational terms (e.g., times as many as) or their corresponding mathematical representations (e.g., proportion), both of which are necessary preconditions of correct modeling solutions (Cummins, Kintsch, Reusser, \& Weimer, 1988; Riley, Greeno, \& Heller, 1983). It is therefore not surprising that solutions based on translation cues are most prevalent in students who have poor text comprehension skills or poor mathematical understanding (Mestre \& Gerace, 1986; Paige \& Simon, 1966).

Although reliance on translation cues has been well documented, little attention has been given to the fact that word problem texts also provide solvers with highly reliable semantic cues. Bassok, Chase, and Martin (1998) examined word problems in a popular textbook series for grades 1 through 8 and found that semantic relations between the objects that appeared in problem texts were highly positively correlated with arithmetic operations that took these objects as arguments. In 97\% of the problems that required addition, the addends were categorically related objects (e.g., red and blue marbles), and in $94 \%$ of the problems that required division, the dividend and the divisor were functionally related objects (e.g., cookies and jars).

According to Bassok et al. (1998), the high correlation between object relations and mathematical operations reflects a structural correspondence between semantic and mathematical relations. Categorically related objects play semantically symmetric roles with respect to their superset (e.g., both red and blue marbles are marbles), just as the addends play mathematically symmetric roles with respect to their sum $(a+b=b+a)$. Similarly, a functionally asymmetric relation between two entities (e.g., cookies are placed in jars rather than vice versa) corresponds to the mathematically asymmetric quotient $(a / b \neq b / a)$. Henceforth, we will designate semantic relations between entities inferred from object sets as either symmetric (categorical) or asymmetric (functional), and we will refer to the corresponding object sets with the terms symmetric (S) and asymmetric (A) sets.

Bassok and her colleagues also found that college students, who have extensive and relatively successful experience with solving word problems, exploit the correlation between semantic and mathematical relations when they construct or solve mathematical word problems (Bassok et al., 1998; Bassok, Wu, \& Olseth, 1995). For example, participants in Bassok et al. (1995) were asked to solve unfamiliar permutation problems that involved random assignment of three objects from one set to another. As one would expect, most participants who attempted to solve these novel problems arrived at incorrect solutions. Interestingly, the mathematical symmetry of their erroneous solutions corresponded to the semantic symmetry of the paired sets. When the problems described A sets (e.g., $n$ computers assigned to $m$ secretaries), most participants placed the numbers representing the paired object sets in mathematically asymmetric structural roles (e.g., $m^{3} / n$ ! or $m / 3 n$ ), but when the problems described S sets (e.g., $n$ doctors from one hospital assigned to work with one of $m$ doctors from another hospital) most participants placed the numbers representing the paired object sets in mathematically symmetric structural roles [e.g., $(m+n) /(m n)^{3}, 3 /(m+n)$ !] . That is, the incorrect mathematical solutions to a permutation problem were structurally analogous to the semantic relation evoked by its paired sets. Bassok and her colleagues referred to the process that mediates the construction of such structurally analogous mathematical solutions with the term semantic alignment.

Both translation and semantic cues are highly associated with correct word problem solutions, and reliance on both types of cues can lead to systematic errors. However, there appears to be a qualitative difference in the conditions and processes that elicit reliance on translation and semantic cues. Translation cues are standardized expressions and thereby allow solvers to circumvent the inferential modeling process and go directly from words to equations (e.g., the keyword times is translated into multiplication). By contrast, semantic cues are not tied to particular words or phrases and require solvers to infer a meaningful relation between the entities in the problem text in order to retrieve an analogous mathematical operation. Furthermore, the logic of semantic alignment is consistent with that of mathematical modeling, in the sense that it establishes a systematic correspondence between a likely situation model and an analogous mathematical model. It is therefore possible that reliance on semantic cues accompanies, rather than circumvents, the modeling process. Put differently, students who exploit semantic cues may be using the inferred object relations in the process of constructing a situation model (e.g., tulips placed in vases), which in turn affects their selection of a structurally analogous mathematical model (e.g., $n$ tulips divided by $m$ vases).

The present study was designed to test the conjecture that, unlike direct-translation solutions, reliance on semantic cues accompanies students' modeling attempts. We reasoned that students rely on semantic cues when they construct a situation model for a given problem. Using mathematical stimuli with object sets that either could or could not help students construct a correct sit- 
uation model, we examined whether students generate a higher proportion of correct mathematical solutions to problems involving the helpful rather than the nonhelpful semantic cues. Note that this differential-accuracy measure of reliance on semantic cues (i.e., semantic effects) depends on students' ability to implement the situation model they construct in the correct mathematical model. It follows that if, as we predict, students rely on semantic cues while engaging in mathematical modeling, semantic effects should be manifest in a task in which they possess sufficient mathematical knowledge but not in one in which they lack sufficient mathematical knowledge.

We tested this prediction by comparing the impact of semantic cues on correct performance in two mathematically isomorphic tasks: (1) solving division word problems (WP) and (2) constructing algebraic equations to represent verbal statements (EQ). The two tasks were matched in their mathematical structure and in the semantic and translation cues they provided, but the participants in our study had significantly more experience with and better mathematical understanding of the WP rather than the EQ task. Both tasks involved identical multiplicative comparison statements, such as "There are 4 times as many cupcakes $(C)$ as brownies $(B)$." As we have mentioned earlier, such statements elicit a high proportion of erroneous direct-translation solutions that reverse the structural roles of the compared sets (i.e., $4 C=B$ instead of $4 B=C$ ). The WP task, which provides a numerical value for the size of set $C$ and presents a problem solving goal, is easier than the EQ task (Wollman, 1983) but elicits similar reversal errors (Lewis \& Mayer, 1987).

As in the previously described studies on semantic alignment effects, the semantic cue manipulation in the present study was the symmetry of the semantic relation between the compared object sets. All of the target problems were mathematically asymmetric, and A sets, such as the functionally related pair students-professors, always supported the correct solution and provided background knowledge supporting the correct relative size of the compared sets (e.g., more students than professors). S sets, such as the categorically related pair cupcakes-brownies, did not support the correct mathematically asymmetric solution and did not provide relevant background knowledge about the relative number of objects in the compared sets. Table 1 presents the matched word problems and statements we used in the present study.

If students use semantic cues as aids to the construction of a situation model, they should be more likely to avoid, or recover from, reversal errors when the problems or statements compare structurally consistent A sets (e.g., students-professors) than when they compare structurally inconsistent S sets (e.g., cupcakes-brownies). Furthermore, because the participants in our study had significantly more mathematical experience with performing the WP than the EQ task, we expected to find more pronounced semantic effects (i.e., higher frequency of correct solutions for A than for S stimuli) in the former than in the latter task.

Our general predictions about the impact of semantic cues on correct mathematical performance in the two tasks depend both on the level of students' text comprehension skills and on the level of their task-relevant mathematical knowledge. In particular, semantic cues should be more likely to affect the construction of a correct situation model for students who have at least some difficulty with text comprehension or with knowledge-based inferences (Kintsch, 1988) than for students who do not have such difficulty. In other words, the semantic cue manipulation should have little impact on the mathematical performance of students who have sufficient text comprehension skills to construct a correct situation model for both A and S stimuli (i.e., ceiling effects of text comprehension skills). Of course, as we have mentioned earlier, the correct-performance measure cannot reveal semantic effects in students who do not possess the relevant mathematical knowledge to implement the situation model they construct (i.e., floor effects of mathematical knowledge). In order to secure a sufficient range of text comprehension skills and of relevant mathematical knowledge that could reveal the impact of semantic cues on correct performance, we examined the magnitude of semantic effects on both tasks in middle school (Grade 7), high school (Grades 9 and 11), and college students.

Because text comprehension skills should improve with grade level, the impact of semantic cues on students' correct performance should decrease with grade. These effects should be further modulated by task-relevant mathematical knowledge. In the WP task, it was presumed that most seventh graders already had the relevant mathematical knowledge (i.e., division solutions) to exhibit semantic effects. Hence, as students in the consecutive grades improve their text comprehension skills, the magnitude of semantic effects in the WP task should decrease with grade. In the EQ task, we did not expect to find significant semantic effects in seventh graders, due to possible floor effects of their algebraic knowledge, or in college students, due to possible ceiling effects of their text comprehension skills. High school students, who were presumed to have intermediate levels of text comprehension skills and mathematical knowledge, should be the most likely group to exhibit semantic effects in the EQ task.

\section{METHOD}

\section{Participants}

Data were collected during the months of April and May over two consecutive years from 816 participants: 184 University of Chicago students; 222 seventh graders at six Chicago parochial schools; and 214 ninth graders and 196 eleventh graders enrolled in algebra courses at one Chicago parochial high school. ${ }^{1}$ Nine students (4 seventh graders, 3 ninth graders, and 2 eleventh graders) who left their test booklets blank were eliminated from the study. In addition, data collected from two middle schools ( $n=18$ and $n=13$ ) were eliminated because students did not return their parent per- 
Table 1

Word Problems and Statements With Semantically Asymmetric and Symmetric Object Sets

Asymmetric Word Problems

At a certain university, there are 3,450 students. There are 6 times as many students as professors. How many professors are there?

Seventy-two commuters join car pools. There are 4 times as many commuters as automobiles. How many automobiles are there?

Asymmetric Statements

At a certain university, there are 6 times as many students as professors.

In a certain car pool, there are 4 times as many commuters as automobiles.

Symmetric Word Problems

On a given day, a certain factory produces 3,450 nails. It produces 6 times as many nails as screws. How many screws does it produce?

One night at Mindy's Restaurant, 72 people ordered cupcakes. Four times as many people ordered cupcakes as brownies. How many people ordered brownies?

Symmetric Statements

A certain factory produces 6 times as many nails as screws.

At Mindy's Restaurant, 4 times as many people ordered cupcakes as brownies.

mission forms in one case or completed more than one test booklet in the other. A random procedure was then used to eliminate observations in each condition in excess of 46 , which was the smallest sample size, occurring in one condition. The final number of participants included in the analyses was 736 . University of Chicago students were paid $\$ 1$ for their participation.

\section{Design and Materials}

A $2 \times 2 \times 4$ design was used in which task (word problem or equation), semantic cue (asymmetric or symmetric), and grade (seventh, ninth, eleventh, or college) were manipulated between subjects. We created two division word problems and two statements with asymmetric object sets (i.e., students and professors, commuters and automobiles) and two division word problems and two statements with symmetric object sets (i.e., cupcakes and brownies, nails and screws). ${ }^{2}$ Asymmetric and symmetric problems and statements were matched for number and translation cue (i.e., times as many). (Again, see Table 1 for the items used.) Booklets were constructed containing two problems or two statements that were matched for the symmetry of the compared sets, with one problem on each page and order of presentation counterbalanced. The cover pages of the booklets instructed the participants either to solve the given word problems or to write equations for the given statements, showing all work. In each grade, students were randomly assigned to one of the four conditions created by crossing semantic symmetry and task.

\section{Procedure}

The procedure used to test middle and high school students was adapted to meet the needs of their instructors. All tests were administered in the students' mathematics class; at the high school and in some classes at four of the middle schools, instructors administered the test to students, and in the rest of the classes at the six middle schools, the first author administered the test. When the experimenter was present, students were allowed as much time as they needed to complete the test (about $10 \mathrm{~min}$ ). Because the test booklets contained few blanks ( $1.5 \%$ of total observations before observations were eliminated), it is presumed that instructors allowed students sufficient time to complete the task. The college students, who were recruited from classes or the student union, were tested either in groups or individually and were allowed as much time as they needed to complete the task.

\section{RESULTS}

Problem solutions and equations were coded as correct if the appropriate arithmetic operation relating the quantities (or variables) was selected, and they were coded as incorrect otherwise. In some cases ( $11 \%$ of the word problems and $4 \%$ of the statements), students gen- erated solutions that they then erased and replaced with a different solution. Only the final solutions were included in the analysis. No solutions were given for a total of two word problems and two statements. These blanks were coded as errors. Because each participant solved two problems or constructed two equations, the participants received scores of 0,1 , or 2 .

Participants' combined scores were submitted to a $2 \times$ $2 \times 4$ analysis of variance (ANOVA) with task (word problem vs. equation), symmetry (asymmetric vs. symmetric), and grade (seventh, ninth, eleventh, or college) as between-subjects variables. Because a Brown-Forsythe median test revealed variance heterogeneity $[F(15,720)=$ $\left.4.35, M S_{\mathrm{e}}=0.540, p<.10\right]$, the significance level was set at .025 . The means of the correct responses in each of the four experimental conditions per grade appear in Figure 1.

The ANOVA produced main effects of task $[F(1,720)=$ $211.71]$, symmetry $[F(1,720)=21.14]$, and grade $[F(3,720)=28.31]\left(M S_{\mathrm{e}}=0.605\right.$ for all $\left.F \mathrm{~s}\right)$. The main effect of task indicates that, consistent with previous findings, students performed better on the WP $(M=$ 1.52) than on the EQ $(M=0.69)$ task. The main effect of symmetry indicates that, as in the previously reported studies on semantic alignments, students were sensitive to the semantic cues and performed better on problems and equations that compared asymmetric $(M=1.24)$ than on those that compared symmetric $(M=0.98)$ object sets. Importantly, and as predicted, this finding is qualified by the significant task $\times$ symmetry interaction $[F(1,720)=17.79]$. Analysis of the simple main effects of this interaction revealed that performance was significantly better on A $(M=1.78)$ than on $\mathrm{S}(M=1.27)$ word problems and equations $[F(1,720)=38.86]$, but there was no difference in performance between the A $(M=0.70)$ and the $\mathrm{S}(M=0.68)$ equations $(p>.025)$. That is, students' sensitivity to semantic cues affected correct performance on a task with which they had sufficient mathematical experience to engage in modeling, but not on a mathematically isomorphic task with which they had relatively little mathematical experience.

The ANOVA also produced a significant task $\times$ grade interaction $[F(3,720)=3.15]$. A trend analysis of this 

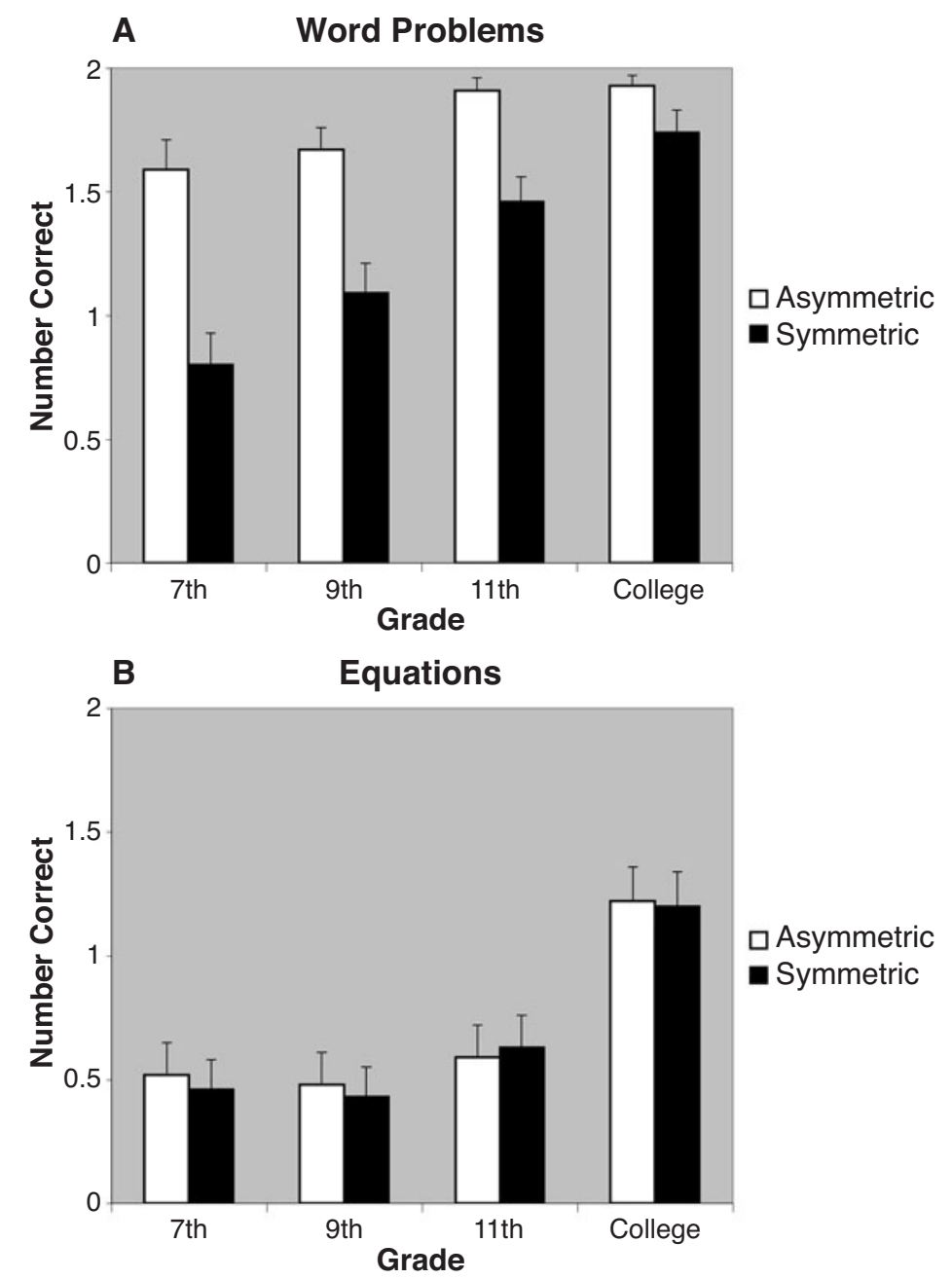

Figure 1. Mean number of correct responses (out of 2) on word problems and equations as a function of semantic symmetry and grade ( $n=46$ per condition).

interaction revealed that performance on word problems improved steadily with grade $[F(1,720)=37.82]$, but performance on equations improved sharply between eleventh grade and college $[F(1,720)=40.58$ for the linear trend; $F(1,720)=15.09$ for the quadratic trend]. The symmetry $X$ grade and the three-way interactions were not significant (both $p \mathrm{~s}>.025$ ), although inspection of Figure 1 suggests that the magnitude of semantic effects in the WP task decreased from seventh grade to college, in agreement with our prediction, but that there were no semantic effects on the EQ task at any grade level. The lack of semantic effects in the performance of high school students on the EQ task is understandable given their low level of correct performance, which was similar to that of seventh graders (i.e., floor effects of algebraic knowledge).

\section{Error Analysis}

In describing the choice of our mathematical tasks, we stated that the most common error in both tasks was a "reversal error" (e.g., $6 S=P$ instead of $6 P=S$, for the students-professors statement). Reversed equations are obviously incorrect mathematical representations of the described situations, and it is unlikely that students who commit these errors engage in mathematical modeling. Nonetheless, given that such errors reverse the variable roles in an otherwise correct mathematical structure, they indicate the existence of at least some algorithmic level of task-relevant mathematical knowledge. The extremely low level of correct performance on the EQ task, which increased only between eleventh grade and college, suggests that a significant proportion of the participants in our study did not possess even an algorithmic level of algebraic knowledge. We therefore distinguished between reversal and all other errors on both tasks and examined whether the relative proportion of reversal errors was higher in the WP than in the EQ task.

The nonreversal errors in the EQ task included expressions that did not contain the equal sign (e.g., " $4 C / A$ ") or expressions on one side of the equal sign (e.g., " $6 S \times$ 
$P=$ "); equations that did not have a multiplicative comparison structure (e.g., " $6 S+P=U$ "; " $N \times S=6$ "); variable assignments (e.g., " $S=36$ and $P=6$ "); and a variety of other incorrect responses (e.g., " $6 N=N$ "; " $6+6=12$ "). Nonreversal errors in the WP task included instantiation of the given (or other) numbers in either a one-step or two-step equation (e.g., "72 - $4=$ 68 "; " $[3,450-6] / 2=1,722$ ") and guesses. Table 2 displays the percentage of reversal errors out of the total number of errors on the first item of the WP and EQ tasks. ${ }^{3}$ Note that, because students' performance was significantly better in the WP than in the EQ task, the number of WP errors (96) in our analysis was much smaller than the number of EQ errors (233).

As can be seen in Table 2, the mean percentage of reversal errors was much higher in the WP task $(80 \%)$ than in the EQ task $(46 \%)\left[\chi^{2}(1, N=329)=31.66, p<.05\right]$. If one considers reversal errors as evidence of some rudimentary, albeit algorithmic, mathematical knowledge of the multiplicative comparison relation, the results above indicate that, consistent with the pattern of correct performance, significantly more participants lacked such knowledge in the EQ than in the WP task. Table 2 also shows that the frequency of reversal errors did not vary with grade in the WP task $\left[\chi^{2}(3, N=96)=4.15, p>\right.$ $.05]$, but it increased with grade in the EQ task $\left[\chi^{2}(3\right.$, $N=233)=32.02, p<.05]$, as more students acquired the algorithmic component of algebraic knowledge that allowed them to represent multiplicative comparison relations as equations.

Examination of the nonreversal errors students made in the EQ task (see examples above) suggests that, lacking even algorithmic algebraic knowledge, students approached the unfamiliar EQ task as if it were an arithmetic WP task with some missing values and performed this task with whatever problem solving skills and mathematical understanding they had at their disposal. Because students from the same population showed sensitivity to semantic cues on the WP task, it is possible that students who performed the EQ task also attempted to construct situation models but aligned them with incorrect mathematical solutions. If so, the structure of their incorrect solutions might reveal semantic effects that were not captured by the correct performance measure.

To examine this possibility, we classified the nonreversal errors by the symmetry of their mathematical structures. Symmetric-structure errors placed the variables in structurally symmetric roles and related the compared variables either by addition (e.g., " $6 N+S=$ $T$ "; " $6 N+S$ ") or by multiplication (" $C \times B=4$ "; " $4 C \times$
$B$ "). Asymmetric-structure errors placed the variables in structurally asymmetric roles. This category included ratios (e.g., " $6 S / P$ "), variable assignments that preserved the relative sizes of the compared sets (e.g., " $P=50$, $S=300$ ") and inequalities (e.g., " $6 S>P$ "). Errors that could not be coded with respect to structural symmetry (e.g., "6 $\times \mathrm{N}=\mathrm{N}$ "; " $\mathrm{C}=, \mathrm{A}=$ "; "C + 4, A - 4") were coded as "other." Although we did not expect semantic symmetry to influence the mathematical structure of WP errors, we included them in the analysis by applying the above coding scheme to the equations underlying the WP solutions.

The distributions of reversal errors and of the symmetric, asymmetric, and "other" nonreversal errors in A and $\mathrm{S}$ word problems and statements are displayed in Table 3. In our analysis of WP errors, we combined all of the nonreversal errors into a single category, because the cell frequencies were quite small. As expected, the mathematical structure of WP errors did not depend on the semantic symmetry of the object sets $\left[\chi^{2}(1, N=\right.$ $96)=1.01, p<.05]$. By contrast, the mathematical structure of EQ errors did depend on the semantic symmetry of the object sets $\left[\chi^{2}(3, N=233)=16.88, p<\right.$ $.05]$. In particular, asymmetric and symmetric mathematical structures interacted with the semantic symmetry of the object sets $\left[\chi^{2}(1, N=233)=13.98, p<.05\right]$. This interaction was due to both the higher percentage of symmetric-structure errors generated for $\mathrm{S}$ than for $\mathrm{A}$ statements (46\% vs. $24 \%$, respectively) and the higher percentage of asymmetric-structure errors generated for A than for S statements (16\% vs. 4\%, respectively). That is, students who lacked even the rudimentary algebraic knowledge to commit reversal errors approached the EQ task as a variant of the familiar WP task and committed structural errors that revealed significant semantic effects.

\section{DISCUSSION}

Bassok et al. (1998) found that semantic relations between objects in the texts of mathematical word problems were highly positively correlated with arithmetic operations that took these objects as arguments: Categorically related objects (e.g., red and blue marbles) were related by addition, whereas functionally related objects (e.g., cookies and jars) were related by division. Bassok and her colleagues also found that college students adhere to this correlation (they engage in "semantic alignments") when they construct or solve mathematical word problems (Bassok et al., 1998; Bassok et al., 1995). The present study was designed to shed light on

Table 2

Percentage of Reversal Errors in the Word Problem and Equation Tasks for Each Grade

\begin{tabular}{|c|c|c|c|c|c|c|c|c|c|c|}
\hline \multirow[b]{2}{*}{ Task } & \multicolumn{2}{|c|}{ Seventh } & \multicolumn{2}{|c|}{ Ninth } & \multicolumn{2}{|c|}{ Eleventh } & \multicolumn{2}{|c|}{ College } & \multicolumn{2}{|c|}{$M$} \\
\hline & $\%$ & $\bar{N}$ & $\%$ & $\bar{N}$ & $\%$ & $\bar{N}$ & $\%$ & $\bar{N}$ & $\%$ & $N$ \\
\hline Word problem & 86 & 42 & 73 & 30 & 87 & 15 & 67 & 9 & 80 & $\overline{96}$ \\
\hline Equation & 23 & 66 & 44 & 70 & 56 & 63 & 79 & 34 & 46 & 233 \\
\hline
\end{tabular}

Note-The final column $(M)$ is the mean percentage over all grades. 
Table 3

Percentage of Reversal Errors and of the Symmetric, Asymmetric, and Other Nonreversal Errors in Asymmetric and Symmetric Word Problems and Statements

\begin{tabular}{lccc}
\hline \multicolumn{1}{c}{ Error Type } & Asymmetric & Symmetric & $M$ \\
\hline \multicolumn{1}{c}{ Word Problems } & \\
Reversal & $(n=22)$ & $(n=74)$ & $(N=96)$ \\
Symmetric & 73 & 82 & 80 \\
Asymmetric & 9 & 7 & 7 \\
Other & 9 & 7 & 7 \\
& 9 & 4 & 5 \\
Reversal & $(n=116)$ & $(n=117)$ & $(N=233)$ \\
Symmetric & 52 & 41 & 46 \\
Asymmetric & 24 & 46 & 35 \\
Other & 16 & 4 & 10 \\
\hline Ner & 9 & 9 & 9 \\
\hline
\end{tabular}

Note-The final column $(M)$ is the mean percentage over symmetry.

the conditions under which students exploit the correlation between object relations and mathematical operations. Specifically, we hypothesized that such semantic effects accompany students' modeling attempts and, therefore, should have more impact on students' performance in a task with which students have more mathematical experience.

In accord with our hypothesis, the correct-performance analysis revealed semantic effects in the WP task, which students performed quite well, but not in the EQ task, which elicited a high proportion of errors. A follow-up error analysis also revealed that most errors in the WP task reversed the variable roles in an otherwise correct mathematical structure and that a high proportion of errors in the EQ task had an incorrect mathematical structure. Interestingly, these structural errors revealed semantic effects in the EQ task that were similar to those found in the erroneous solutions generated by college students who solved unfamiliar permutation word problems in Bassok et al. (1995). The semantic effects revealed in the structural errors students made in the EQ task appear to be contributed by those students who performed this unfamiliar task as if it were a missing-value variant of the familiar WP task. That is, students with no knowledge of algebra transferred their extensive modeling experience from the familiar and better understood WP task to the novel EQ task.

Both the correct-performance and error-structure results pertaining to semantic effects suggest that such effects were more pronounced in younger students. However, the correct-performance measure did not yield a statistically significant interaction of semantic effects with grade, and the number of structural errors was insufficient for an analysis of the interaction of semantic effects with grade. The only statistically significant effects involving grade level pertained to changes in students' mathematical understanding of the two tasks. In the WP task, students' performance improved gradually with grade, but in the EQ task, students' performance improved only between eleventh grade and college.
The error analysis revealed a more gradual learning curve in the EQ task, as students transitioned from structural (e.g., " $6 S+P=U$ ") to reversal (e.g., " $6 S=P$ ") errors. Although this transition from arithmetic to algebra indicates some advancement in students' mathematical knowledge, the high proportion of reversal errors in the EQ task cannot be taken as evidence of mathematical understanding. As argued by Greeno (1987), it appears that the algebraic knowledge students initially acquire is algorithmic in nature and lacks the modeling component. It is quite interesting that students who had no knowledge of algebra spontaneously transferred their modeling skill from the familiar WP task to the novel EQ task, whereas those with some algebra training failed to realize both the similarity between the two tasks and the relevance of their previously acquired modeling skill to the task of constructing algebraic equations. The discrepancy between students' manifestation of good modeling skills in the WP task and algorithmic knowledge in the EQ task is consistent with research in mathematics education showing that students' initial algebraic knowledge is conceptually disconnected from their arithmetic knowledge (e.g., Herscovics \& Linchevski, 1994; Kieran, 1992).

To summarize, it appears that semantic cues affect the interpretive text comprehension stage of the modeling process, whereas students' mathematical understanding of the target task determines whether or not they engage in mathematical modeling and, if they do, how they implement the situation model they have constructed. The familiar and better understood WP task elicited semantic effects at all grade levels, the impact of semantic cues on students' performance being reflected in higher proportions of correct solutions to problems with structurally consistent (A) rather than structurally inconsistent (S) object sets. The less familiar (or unfamiliar) and poorly understood EQ task elicited semantic effects only for students who, having no relevant algebraic knowledge, approached this task as if it were a variant of the more familiar problem solving task. The impact of semantic cues on the performance of these students was reflected in the mathematical structure of their erroneous solutions. Finally, students who acquired the most rudimentary algorithmic variant of algebraic knowledge constructed reversed multiplicative comparison equations and did not exhibit any semantic effects.

Although the present results support our entering conjecture that students exploit semantic cues when they engage in mathematical modeling, our data cannot illuminate the process by which students' modeling attempts interact with their reliance on semantic cues. In particular, it is possible that semantic cues always affect the way students understand mathematical texts, but that the impact of such interpretive effects is subsequently modulated by students' mathematical knowledge and by their decision about whether or not to engage in modeling. Alternatively, students' mathematical understanding and 
modeling decisions could precede the text comprehension stage, leading them to take differential notice of semantic cues. A study that compares the reading time of multiplicative comparison statements with semantically symmetric and asymmetric object sets in the problem solving and equation construction tasks could probably distinguish between these possibilities. Of course, it remains unclear to what extent students' modeling attempts and their reliance on semantic cues are intentional or reflect some form of implicit knowledge.

Irrespective of the specific mechanism that mediates students' reliance on semantic cues, the results of the present study clearly show that the conditions that elicit semantic effects are orthogonal to those that elicit reliance on translation cues. Reliance on translation cues (e.g., times implies multiplication) is more characteristic of students who possess only algorithmic knowledge of the target task and who circumvent the interpretive process of mathematical modeling. By contrast, reliance on semantic cues (e.g., the relation between students and professors is functionally asymmetric and usually corresponds to division) is characteristic of students who have a more advanced understanding of the target task and who attempt to engage in the sense-making activity of mathematical modeling. Taken together, these findings may have important implications for mathematics educators. For example, by varying the objects in the texts of mathematical word problems or mathematical statements, educators could establish whether or not students attempt to engage in mathematical modeling. This easyto-implement diagnostic tool could assist mathematics educators in identifying students who need help with understanding how the formal rules of mathematics should be used to model real-life situations.

\section{REFERENCES}

Baranes, R., Perry, M., \& Stigler, J. W. (1989). Activation of realworld knowledge in the solution of word problems. Cognition \& Instruction, 6, 287-318.

Bassok, M., Chase, V. M., \& Martin, S. A. (1998). Adding apples and oranges: Alignment of semantic and formal knowledge. Cognitive Psychology, 35, 99-134.

BAssoK, M., Wu, L. L., \& Olseth, K. L. (1995). Judging a book by its cover: Interpretative effects of content on problem-solving transfer. Memory \& Cognition, 23, 354-367.

Clement, J. (1982). Algebra word problem solutions: Thought processes underlying a common misconception. Journal for Research in Mathematics Education, 13, 16-30.

Clement, J., Lochhead, J., \& Soloway, E. (1979). Translating between symbol systems: Isolating a common difficulty in solving algebra word problems (Tech. Rep. No. 79-19). Amherst, MA: University of Massachusetts, Department of Physics and Astronomy.

Cummins, D. D., Kintsch, W., Reusser, K., \& Weimer, R. (1988). The role of understanding in solving word problems. Cognitive Psychology, 20, 405-438.

ENGLISH, L. D. (1997). Children's reasoning processes in classifying and solving computational word problems. In L. D. English (Ed.), Mathematical reasoning: Analogies, metaphors, and images (pp. 191220). Mahwah, NJ: Erlbaum.

ENGlish, L. D., \& Halford, G. S. (1995). Mathematics education: Models and processes. Mahwah, NJ: Erlbaum.
Greeno, J. G. (1987). Instructional representations based on research about understanding. In A. Schoenfeld (Ed.), Cognitive science and mathematics education (pp. 61-88). Hillsdale, NJ: Erlbaum.

GreER, B. (1993). The mathematical modeling perspective on wor(1)d problems. Journal of Mathematical Behavior, 12, 239-250.

Herscovics, N., \& LinchevsKi, L. (1994). A cognitive gap between arithmetic and algebra. Educational Studies in Mathematics, 27, 5978.

Hinsley, D. A., Hayes, J. R., \& Simon, H. A. (1977). From words to equations: Meaning and representation in algebra word problems. In M. A. Just \& P. A. Carpenter (Eds.), Cognitive processes in comprehension (pp. 89-106). Hillsdale, NJ: Erlbaum.

Jenson, D. R., Beus, G. B., \& Storm, G. (1968). Simultaneous statistical tests on categorical data. Journal of Experimental Education, 36, 46-55.

KiERAN, C. (1992). The learning and teaching of school algebra. In D. A. Grouws (Ed.), Handbook of research on mathematics teaching and learning (pp. 371-389). New York: Macmillan.

KInTSCH, W. (1988). The role of knowledge in discourse comprehension: A construction-integration model. Psychological Review, 95, 163-182.

LEWIS, A. B., \& MAYER, R. E. (1987). Students' miscomprehension of relational statements in arithmetic word problems. Journal of Educational Psychology, 79, 363-371.

Mestre, J., \& Gerace, W. (1986). The interplay of linguistic factors in mathematical translation tasks. Focus on Learning Problems in Mathematics, 8, 59-72.

Nesher, P., \& Teubal, E. (1975). Verbal cues as an interfering factor in verbal problem solving. Educational Studies in Mathematics, 6, 41-51.

Paige, J. M., \& Simon, H. A. (1966). Cognitive processes in solving algebra word problems. In B. Kleinmutz (Ed.), Problem solving: Research, method, and theory (pp. 51-119). New York: Wiley.

Reusser, K. (1988). Problem solving beyond the logic of things: Contextual effects on understanding and solving word problems. Instructional Science, 17, 309-338.

Riley, M. S., Greeno, J. G., \& Heller, J. I. (1983). Development of children's problem-solving ability in arithmetic. In H. P. Ginsburg (Ed.), The development of mathematical thinking (pp. 153-196). San Diego: Academic Press.

Verschaffel, L., De Corte, E., \& Lasure, S. (1994). Realistic considerations in mathematical modeling of school arithmetic word problems. Learning \& Instruction, 4, 273-294.

Wollman, W. (1983). Determining the source of error in a translation from sentence to equation. Journal for Research in Mathematics Education, 14, 169-181.

\section{NOTES}

1. Middle schools with students from a similar socioeconomic background that was also similar to that of the high school participants were identified with the help of a principal at one of the participating schools. Because the participating high school and middle schools were not preparatory schools, the mathematical ability of the students from these schools may not have been comparable to that of the University of Chicago students. Hence, differences in performance between the college and secondary students in our sample will, to some degree, reflect ability as well as experience.

2. In midstudy, we substituted the pair "cupcakes and brownies" for the pair "cheesecakes and strudels" because some students were not familiar with the word strudel.

3. To preserve the chi-square test's assumption of independence, we coded only the errors committed on the first problem solved. Because we conducted multiple tests on the samples of errors from the WP and EQ tasks, the obtained chi-square values were compared with the critical value of the Bonferroni chi square (Jenson, Beus, \& Storm, 1968).

(Manuscript received October 2, 2002; revision accepted for publication June 18, 2004.) 